\title{
APLICAÇ̃̃O DE UM MODELO FUZZY DEA PARA PRIORIZAR MODOS DE FALHA EM SISTEMAS NUCLEARES
}

\author{
Pauli Adriano de Almada Garcia* \\ Departamento de Administração / PUVR \\ Universidade Federal Fluminense (UFF) \\ Volta Redonda - RJ, Brasil \\ pauliadriano@gmail.com \\ P. F. Frutuoso e Melo \\ R. Schirru \\ Programa de Engenharia Nuclear / COPPE \\ Universidade Federal do Rio de Janeiro (UFRJ) \\ Rio de Janeiro - RJ, Brasil \\ frutuoso@con.ufrj.br \\ schirru@lmp.ufrj.br \\ * Corresponding author / autor para quem as correspondências devem ser encaminhadas \\ Recebido em 08/2007; aceito em 03/2009 após 1 revisão \\ Received August 2007; accepted March 2009 after one revision
}

\section{Resumo}

Neste artigo apresenta-se a aplicação de um modelo híbrido, baseado na análise envoltória de dados e em conjuntos fuzzy, para estabelecer uma priorização dos modos de falha levantados numa análise dos modos e efeitos de falha. O objetivo principal é ilustrar a aplicabilidade da modelagem para analisar sistemas complexos, como o sistema de controle químico e volumétrico de uma usina nuclear do tipo água pressurizada, onde uma grande quantidade de modos de falha deve ser avaliada. $\mathrm{O}$ modelo apresentado possibilita tratar variáveis linguísticas, i.e., permite que se tratem as opiniões de especialistas de modo mais efetivo num contexto de análise de riscos industriais quando há poucos dados disponíveis, como pode ocorrer em projetos com inovação tecnológica. Os resultados obtidos demonstram o potencial e a viabilidade de se utilizar uma modelagem híbrida para se estabelecer uma ordem de prioridade entre os diferentes modos de falha associados a um sistema complexo.

Palavras-chave: CVCS; FMEA; DEA; opinião de especialistas; número de priorização de riscos.

\begin{abstract}
In this paper one presents a data envelopment analysis and fuzzy sets based hybrid model application, in order to establish a priority ranking for the failure modes evaluated in a failure mode and effect analysis. The main objective is to present the applicability of this hybrid model to analyze complex systems, as the chemical and volumetric control system of a pressurized water reactor power plant, where a great bunch of failure modes must typically be evaluated. The presented modeling makes it possible the consideration of linguistic variables, i.e., it permits that one considers most effectively expert opinion in a risk analysis context when scarce data is available, as may occur in the plant design phase. The results obtained demonstrated the powerfulness and the viability of use of the proposed hybrid modeling to establish a priority ranking among many failure modes concerning a complex system.
\end{abstract}

Keywords: CVCS; FMEA; DEA; expert opinion; risk priority number. 


\section{Introdução}

Com os avanços científicos e tecnológicos, cada vez mais têm se desenvolvido técnicas de apoio à tomada de decisão onde a quantidade de informações específicas para o sistema sob análise não é suficiente para a realização de inferências estatísticas e a opinião de especialistas é o fator preponderante. Em outras palavras, cada vez mais a experiência de especialistas vem sendo requisitada para substanciar análises de riscos em instalações industriais, principalmente quando se está envolvido com tecnologias de ponta, como é o caso das usinas termonucleares (Ayyub, 2001).

Para o funcionamento seguro das usinas nucleares, estão disponíveis diferentes sistemas de segurança. Dentre estes sistemas, pode-se destacar o sistema de controle químico e volumétrico ("Chemical and Volumetric Control System" - CVCS). O CVCS é projetado para atender ao sistema de refrigeração do reator ("Reactor Coolant System" - RCS), fornecendo-lhe os seguintes serviços:

i) Manutenção do nível de água programado no pressurizador;

ii) Manutenção dos selos das bombas de injeção do RCS;

iii) Controle das condições químicas da água de refrigeração do reator;

iv) Provimento de meios para o enchimento, drenagem e testes de pressão do RCS.

Diante destas informações, pode-se então perceber a importância de se garantir o bom funcionamento do CVCS. Estão disponíveis diferentes estudos associados a este sistema, sendo o principal deles associado a usinas a água pressurizada ("Pressurized Water Reactor" - PWR) [2], onde são avaliados os efeitos do envelhecimento de tal sistema.

Um dos estudos mais importantes é a análise dos modos e efeitos de falha ("Failure Mode and Effect Analysis" - FMEA) que é uma técnica importante de auxilio à identificação de modos de falha críticos e estabelecimento de recomendações para melhorias (EPRI, 1996; IAEA, 2001 e IAEA, 2002).

No presente artigo, apresenta-se uma abordagem onde são combinadas as técnicas baseadas em análise envoltória de dados ("Data Envelopment Analysis"-DEA) (Charnes et al., 1978) e lógica fuzzy (Zadeh, 1987). Estas técnicas são utilizadas para estabelecer uma análise comparativa não-paramétrica, entre os modos de falha, onde possam ser consideradas as variáveis linguísticas, proveniente da opinião de especialistas (Garcia, 2006).

$\mathrm{O}$ grande diferencial da abordagem a ser apresentada neste artigo para as demais utilizadas na priorização de modos de falha, considerando variáveis linguísticas, é o fato de no presente caso não ser necessário o desenvolvimento de regras de inferência especialistas. Este fato contribui sobremaneira ao desenvolvimento da FMEA, tendo em vista que os especialistas envolvidos deverão fornecer unicamente a representação das variáveis linguísticas a serem utilizadas na caracterização dos índices. Esta representação é feita por meio das funções de pertinência (ou de possibilidades).

Para as abordagens baseadas em sistemas de inferência do tipo Mamdani, além da representação, por meio das funções de pertinência, das variáveis linguísticas utilizadas, devem ser estabelecidas as regras especialistas de inferência para, posteriormente, serem levantados os índices "crisp" para serem utilizados como variáveis de entrada no processo de inferência (Lapa \& Guimarães, 2004; Bowles \& Peláez, 1995).

Para demonstrar a viabilidade de se utilizar a abordagem proposta em sistemas complexos, assim como suas respectivas vantagens em relação às demais abordagens baseadas em 
sistemas de inferência fuzzy (Lapa \& Guimarães, 2004; Pillay \& Wang, 2003; Peláez \& Bowles, 1996; Xu et al., 2002) ou análise envoltória de dados unicamente (Garcia, 2001; Garcia et al., 2002), será apresentado um estudo de caso associado ao CVCS, e os resultados obtidos serão discutidos.

\section{Apresentação do sistema de controle químico e volumétrico}

Os subsistemas tipicamente incluídos neste sistema são (Lapa \& Guimarães, 2004):

- Carga e descarga do sistema de selagem com água;

- Purificação e controle químico do refrigerante;

- Controle do sistema de reserva;

- Sistema de regeneração térmica do boro.

Muitos dos componentes do CVCS estão localizados na parte do prédio da contenção da usina. Devido a isto, a degradação do sistema contribui sobremaneira para a liberação para a atmosfera de materiais radioativos. Para melhor compreender os efeitos associados à falha do CVCS, é preciso que se separem os seus modos de operação em: partida, normal e desligamento.

O CVCS para usinas do tipo PWR possui funções de operação tanto para situações normais quanto para situações de acidente (Grove \& Travis, 1995). Em operação normal, as três funções básicas são: (i) purificar o reator; (ii) controlar a composição do refrigerante e (iii) suprir as bombas de refrigeração do reator.

Em situações de emergência, as funções primárias são: (i) prover alta pressão de injeção de segurança; (ii) suprir as bombas de refrigeração do reator; (iii) aumentar o nível de boro do refrigerante.

\section{Apresentação da metodologia}

Um processo de produção pode ser avaliado considerando-se duas características de eficiência: técnica e econômica. A eficiência técnica é a proficiência com que os insumos utilizados no processo de produção são convertidos em produtos. Já a eficiência econômica refere-se à otimização de custos e lucros. O processo produtivo é dito eficiente se não existir outro alternativo, ou uma combinação de processos, que produza a mesma quantidade com custos menores.

A eficiência técnica diz respeito ao aspecto físico da produção, enquanto que a econômica é uma extensão da técnica, ou seja, envolve aspectos físicos e monetários. Com isto, o processo para ser economicamente eficiente requer a máxima eficiência técnica (De Souza, 2003).

A eficiência técnica é orientada para o produto ou para o insumo, isto é:

- Qual é a máxima quantidade de produtos que se pode produzir tendo-se fixado uma dada quantidade de insumos? (pergunta feita quando se olha para os produtos);

- Qual é a quantidade mínima de insumo que se pode utilizar para gerar uma dada quantidade de produtos? (pergunta feita quando se olha para os insumos).

O ótimo, para ambos os casos, pode ser estimado em relação a uma situação ideal, por exemplo, estima-se a função de produção e calcula-se a renda líquida máxima, que é a base

Pesquisa Operacional, v.29, n.2, p.383-402, Maio a Agosto de 2009 
de comparação. Ou ainda, estima-se a função custo e calcula-se o custo mínimo, em relação ao qual o custo observado é avaliado.

Esse ótimo pode ser calculado para cada grupo de produtores e, com isto, um produtor é comparado com os que produzem tanto quanto ou mais do que ele, com quantidade de insumos igual ou menor. Com este procedimento, alcança-se a eficiência relativa.

A avaliação por meio de fronteiras é a base das estimativas de eficiência. Este tipo de estudo teve início com Farrel em 1957, que propôs um modelo empírico para a medida de eficiência relativa, em oposição ao modelo de função de produção. Segundo Farrel (1957), é melhor determinar a medida da eficiência da empresa em relação ao melhor nível de eficiência observado do que em relação a algum ideal inatingível. Neste caso, a fronteira de eficiência é construída pelos valores observados de insumos e de produtos, e não por valores estimados.

Farrel propôs um método econométrico para estimar a função de produção e para medir a eficiência técnica, que é um método não-paramétrico de fronteira determinística.

Seja uma empresa que produz y com os insumos $x_{1}$ e $x_{2}$, com uma fronteira de produção seja dada por $y=f\left(x_{1}, x_{2}\right)$. Sob retorno constante de escala, pode-se escrever $1=f\left(x_{1}, x_{2}\right)$. Expressando $x_{2}$ em função de $x_{1}$, obtém-se a isoquanta unitária, que é a fronteira técnica.

$\mathrm{Na}$ Figura 1, Isoq $\mathrm{x}$ é a isoquanta unitária da empresa eficiente, ou seja, é a melhor combinação de insumos que podem produzir no máximo y. Na prática, essa isoquanta é desconhecida, sendo necessário estimar a função de produção da empresa eficiente. Se outra empresa usa a quantidade de insumos definida pelo ponto $\mathrm{P}$ para produzir a mesma quantidade de produto, sua eficiência técnica é representada pela distância QP. Esta quantidade QP indica o quanto os insumos podem ser proporcionalmente reduzidos sem diminuir a produção. A razão QP/OP mede a ineficiência técnica. Sendo assim, a eficiência técnica é dada por:

$$
\mathrm{ET}=1-\frac{\mathrm{QP}}{\mathrm{OP}}
$$

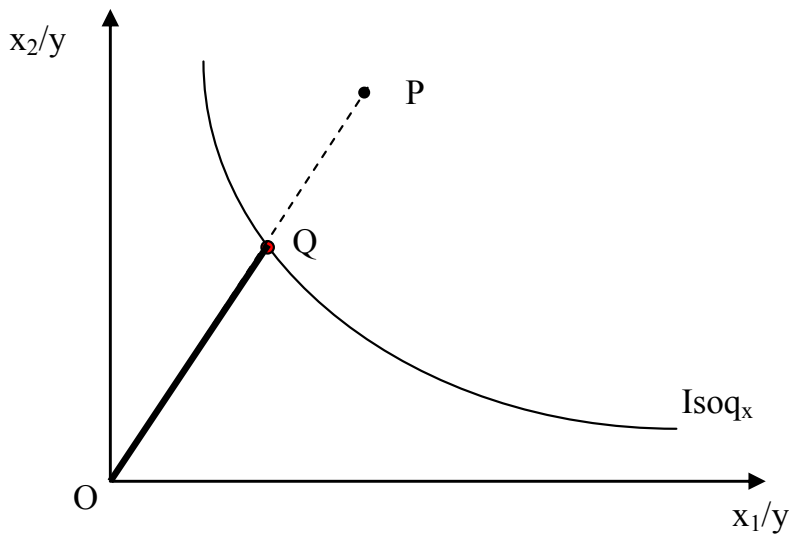

Figura 1 - Isoquanta segundo a ótica dos insumos.

Neste caso, se ET $=1$ significa que a empresa é tecnicamente eficiente, pois está sobre a isoquanta eficiente, como é o caso do ponto Q (o ponto $\mathrm{P}$ coincidirá com o ponto Q).

Foi a partir destes conceitos que se desenvolveram os modelos de análise envoltória de dados. 


\subsection{Modelagem DEA}

Os modelos econométricos paramétricos, tanto estocásticos quanto determinísticos, impõem formas funcionais para representar as tecnologias. A abordagem baseada em envoltória de dados (DEA) não necessita desta hipótese. As eficiências das unidades de tomada de decisões ("Decision Making Unitis" - DMU) são medidas em relação à fronteira, que é gerada pelos vetores (x,y), onde x é o vetor de insumos (doravante denominados inputs) e y é o vetor de produtos (doravante denominados outputs). É importante ressaltar que no presente caso a fronteira é gerada e não estimada.

Com base nos conceitos de Farrel (1957), Charnes, Cooper \& Rhodes (1978) deram início à abordagem não paramétrica para serem considerados múltiplos inputs e outputs. O modelo por eles proposto, comumente denominado de CCR devido às inicias de seus respectivos nomes, é dado por:

$$
\operatorname{Max} \mathrm{h}_{0}=\mathrm{v}^{\mathrm{t}} \mathrm{y}_{0}
$$

sujeito a:

$$
\begin{aligned}
& \mathrm{u}^{\mathrm{t}} \mathrm{x}_{0}=1 \\
& \mathrm{v}^{\mathrm{t}} \mathrm{Y}-\mathrm{u}^{\mathrm{t}} \mathrm{X} \leq 0, k=1, \ldots, n \\
& \mathrm{u}, \mathrm{v} \geq 0
\end{aligned}
$$

onde:

$\mathrm{h}_{\mathrm{o}}$ : é a eficiência da $\mathrm{DMU}_{\mathrm{o}}$;

$v^{\text {t: }}$ são os pesos associados aos outputs;

$\mathrm{u}^{\mathrm{t}}$ : são os pesos associados aos inputs;

$\mathrm{y}_{0}$ : vetor com os níveis dos outputs da $\mathrm{DMU}_{0}$;

$\mathrm{x}_{0}$ : vetor com os níveis dos inputs da $\mathrm{DMU}_{0}$;

$\mathrm{X}$ : Matriz com os níveis dos inputs das demais DMU;

Y: Matriz com os níveis dos outputs das demais DMU.

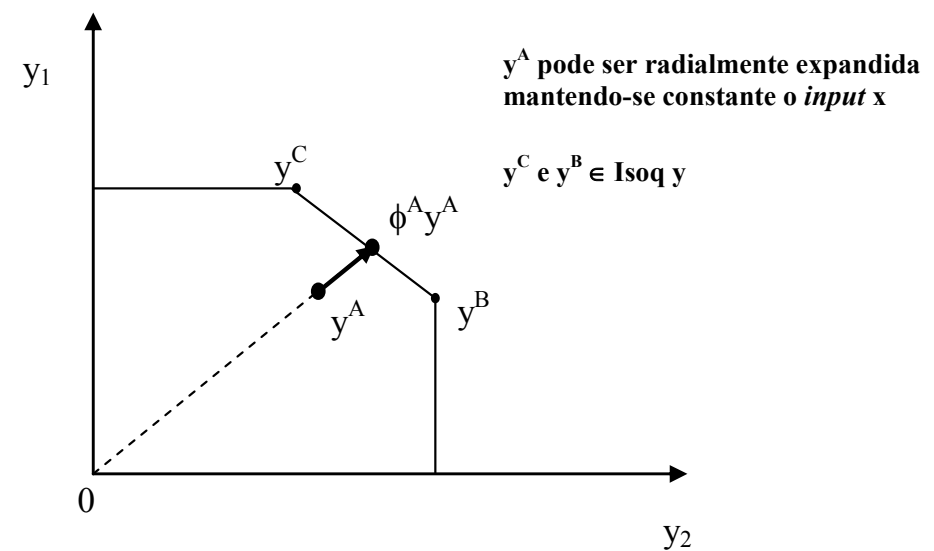

Figura 2 - Medida de eficiência segundo Dabreu e Farrel. 
De modo a medir a eficiência de cada uma das unidades de tomada de decisão levantadas, uma fronteira de eficiência é estabelecida com os dados observados de cada DMU. Todas as DMU envoltas por esta fronteira terão suas eficiências medidas de forma semelhante à apresenta na Figura 1, sendo que no presente caso ter-se-á uma combinação entre múltiplos inputs e outputs, conforme ilustrado na Figura 2, que é uma representação da medida de eficiência de Dabreu e Farrel (apud Farrel, 1957).

A teoria de conjuntos fuzzy vem sendo usada como técnica para quantificar dados imprecisos ou vagos nos modelos DEA. Os modelos fuzzy DEA são baseados nos modelos de programação linear fuzzy. O modelo CCR com coeficientes fuzzy (FCCR) apresenta-se da seguinte forma (Lertworasirikul, 2003):

$$
\begin{aligned}
& \text { Max } v^{\mathrm{T}} \tilde{y}_{0} \\
& \text { sujeito a : } \\
& \mathrm{u}^{\mathrm{T}} \tilde{\mathrm{X}}_{0}=1 \\
& \mathrm{v}^{\mathrm{T}} \tilde{\mathrm{Y}}-\mathrm{u}^{\mathrm{T}} \tilde{\mathrm{X}} \leq 0 \\
& \mathrm{u}, \mathrm{v} \geq 0
\end{aligned}
$$

onde:

$\tilde{\mathrm{x}}_{0}$ é o vetor coluna das variáveis fuzzy input da $\mathrm{DMU}_{0}$;

$\tilde{\mathrm{X}}$ : é a matriz das variáveis fuzzy input das demais DMU;

$\tilde{y}_{0}$ : é o vetor coluna das variáveis fuzzy output da $\mathrm{DMU}_{0}$;

$\tilde{Y}$ : é a matriz das variáveis fuzzy output das demais DMU.

De forma similar ao modelo CCR tradicional, as restrições $u^{T} \tilde{x}_{0}=1$ e $v^{T} \tilde{Y}-u^{T} \tilde{X} \leq 0$ no modelo FCCR são utilizadas para normalizar o valor de $v^{T} \tilde{y}_{0}$. Entretanto, a função objetivo pode exceder 1, visto que a primeira e a segunda restrições na formulação (6) são satisfeitas possibilisticamente. Isto é, visto que os parâmetros são conjuntos fuzzy, as restrições são aproximadamente iguais ou menores ou iguais a um e zero, respectivamente.

Tendo em vista que se está considerando coeficientes fuzzy, o modelo CCR não pode mais ser resolvido utilizando-se um algoritmo padrão para a resolução tradicional de problemas de programação linear. Com variáveis fuzzy de entrada e saída, as condições das soluções ótimas para o modelo CCR crisp precisam ser esclarecidas e generalizadas. O modelo CCR fuzzy correspondente é geralmente resolvido utilizando-se algum método de ordenação para conjuntos fuzzy. Algumas das principais publicações referentes à resolução de modelos fuzzy DEA podem ser categorizadas de quatro maneiras: i) as que enfocam o conceito de tolerância (Sengupta, 1992; Kahraman \& Tolga, 1998), ii) os enfoques baseados em desfuzzificação (Lertworasirikul, 2001), iii) os enfoques baseados em graus de pertinência (Maeda et al., 1998; Kao \& Liu, 2000; Lertworasirikul, 2001) e iv) os enfoques baseados em ordenação de números fuzzy (Guo \& Tanaka, 2001). Segundo Lertworasirikul (2003), para cada um dos enfoques supracitados têm-se prós e contras no que se refere ao trato das incertezas associadas aos dados. Outras abordagens para os casos em que se tenham restrições de igualdade podem ser vistas em Galperin \& Ekel (2005). Abordagens baseadas em otimização discreta aplicada na resolução de problemas com variáveis fuzzy podem ser vistas em Ekel \& Schuffner Neto (2006).

No presente trabalho, pretende-se utilizar um modelo baseado em teoria de possibilidades para solucionar o problema de programação linear com coeficientes fuzzy apresentado em 
Kahraman \& Tolga (1998) e Lertworasirikul (2001). Nesta abordagem o problema de programação linear é resolvido, via método simplex, para diferentes níveis de possibilidades para o atendimento das restrições. A fundamentação teórica referente ao desenvolvimento deste modelo é apresentada na seção a seguir.

\subsubsection{Modelagem fuzzy DEA de possibilidades}

Para a modelagem via teoria de possibilidades o que se faz é considerar os eventos fuzzy por meio de medidas de possibilidades. Para tanto, introduzem-se alguns conceitos referentes à teoria de possibilidades.

\subsubsection{Teoria das possibilidades}

Seja $\left(\Theta_{i}, \wp\left(\Theta_{i}\right), \pi_{i}\right)$, para cada $i=1,2 \ldots, n$, um espaço de possibilidades com $\Theta_{i}$ sendo um conjunto não-vazio de interesse; $\wp\left(\Theta_{\mathrm{i}}\right)$ a coleção de todos os subconjuntos de $\Theta_{\mathrm{i}}$; e $\pi_{\mathrm{i}} \in[0,1]$ uma medida de possibilidade para cada elemento de $\wp\left(\Theta_{\mathrm{i}}\right)$.

Dado um espaço de possibilidades conforme o supracitado e com:

i. $\pi(\varnothing)=0, \pi\left(\Theta_{\mathrm{i}}\right)=1$; e

ii. $\pi\left(\cup_{\mathrm{i}} A_{\mathrm{i}}\right)=\sup \left\{\pi\left(A_{\mathrm{i}}\right)\right\}$ com cada $A_{\mathrm{i}} \in \wp\left(\Theta_{\mathrm{i}}\right)$.

Zadeh (1978) definiu uma variável fuzzy, $\tilde{\xi}$, como uma função real definida em $\Theta_{\mathrm{i}}$ com função de pertinência:

$$
\mu_{\tilde{\xi}}(s)=\pi\left(\left\{\theta_{i} \in \Theta_{i} \mid \tilde{\xi}\left(\theta_{i}\right)=s\right\}\right)=\sup _{\theta_{i} \in \Theta_{i}}\left\{\pi\left(\left\{\theta_{i}\right\}\right) \mid \tilde{\xi}\left(\theta_{i}\right)=s\right\}, \forall s \in \mathfrak{R}
$$

Considerando-se um produto no espaço de possibilidades supracitado tal que $\Theta=\Theta_{1} \times \Theta_{2} \times \Theta_{3} \ldots \times \Theta_{\mathrm{n}}$, onde $\times$ representa um produto cartesiano, e da teoria de possibilidades,

$$
\pi(A)=\min _{\mathrm{i}=1,2, \ldots, \mathrm{n}}\left\{\pi_{\mathrm{i}}\left(\mathrm{A}_{\mathrm{i}}\right) \mid \mathrm{A}=\mathrm{A}_{1} \times \mathrm{A}_{2} \times \ldots \times \mathrm{A}_{\mathrm{n}}, \mathrm{A}_{\mathrm{i}} \in \wp\left(\Theta_{\mathrm{i}}\right)\right\}
$$

Sejam $\tilde{a}$ e $\tilde{b}$ duas variáveis fuzzy dos espaços de possibilidades $\left(\Theta_{1}, \wp\left(\Theta_{1}\right), \pi_{1}\right)$ e $\left(\Theta_{2}, \wp\left(\Theta_{2}, \pi_{2}\right)\right.$, respectivamente. Com isto, $\tilde{a} \leq \tilde{b}$ é um evento fuzzy definido no espaço de possibilidades do produto $\left(\Theta=\Theta_{1} \times \Theta_{2}, \wp(\Theta), \pi\right)$, com:

$$
\pi(\tilde{a} \leq \tilde{b})=\sup _{\substack{\theta_{1} \in \Theta_{1} \\ \theta_{2 \in} \Theta_{2}}}\left\{\pi\left(\left(\theta_{1}, \theta_{2}\right) \mid \tilde{a}\left(\theta_{1}\right) \leq \tilde{b}\left(\theta_{2}\right)\right)\right\}=\sup _{\substack{\theta_{1} \in \Theta_{1} \\ \theta_{2 \in} \Theta_{2}}}\left\{\min \left\{\pi\left(\theta_{1}\right), \pi\left(\theta_{2}\right)\right\} \mid \tilde{a}\left(\theta_{1}\right) \leq \tilde{b}\left(\theta_{2}\right)\right\}
$$

Combinando a equação (9) com a definição de variável fuzzy (7) tem-se:

$$
\pi(\tilde{a} \leq \tilde{b})=\sup _{s, t \in \mathfrak{R}}\left\{\min \left(\mu_{\tilde{a}}(s), \mu_{\tilde{b}}(t)\right) \mid s \leq t\right\}
$$

De modo similar, as possibilidades dos eventos fuzzy do tipo $\tilde{a} \leq \tilde{b}$ e $\tilde{a}=\tilde{b}$ definidos no espaço de possibilidades $(\Theta, \wp(\Theta), \pi)$ são: 


$$
\begin{aligned}
& \pi(\tilde{a}<\tilde{b})=\sup _{s, t \in \Re}\left\{\min \left(\mu_{\tilde{a}}(s), \mu_{\tilde{b}}(t)\right) \mid s<t\right\} \\
& \pi(\tilde{a}=\tilde{b})=\sup _{s, t \in \mathfrak{R}}\left\{\min \left(\mu_{\tilde{a}}(s), \mu_{\tilde{b}}(t)\right) \mid s=t\right\}
\end{aligned}
$$

respectivamente.

Para o caso em que $\tilde{b}$ seja um número crisp b, então as possibilidades dos correspondentes eventos fuzzy serão dadas por:

$$
\begin{aligned}
& \pi(\tilde{a} \leq b)=\sup _{s, t \in \mathfrak{R}}\left\{\min \left(\mu_{\tilde{a}}(s)\right) \mid s \leq b\right\} \\
& \pi(\tilde{a}<b)=\sup _{s, t \in \mathscr{R}}\left\{\min \left(\mu_{\tilde{a}}(s)\right) \mid s<b\right\} \\
& \pi(\tilde{a}=b)=\mu_{\tilde{a}}(b)
\end{aligned}
$$

Sejam $\tilde{a}_{1}, \tilde{a}_{2}, \ldots, \tilde{a}_{n}$ variáveis fuzzy, e seja $f_{\mathrm{j}}: \mathrm{R}^{\mathrm{n}} \rightarrow \mathrm{R}$ uma função real, para $\mathrm{j}=1, \ldots, \mathrm{m}$. A possibilidade de um evento fuzzy $f_{\mathrm{j}}\left(\tilde{a}_{1}, \tilde{a}_{2}, \ldots, \tilde{a}_{n}\right) \leq \mathrm{b}, \mathrm{j}=1, \ldots, \mathrm{m}$, é dada por:

$$
\pi\left(f_{j}\left(\tilde{a}_{1}, \tilde{a}_{2}, \ldots, \tilde{a}_{n}\right) \leq b, j=1, \ldots, m\right)=\sup _{s_{1}, s_{2}, \ldots s_{n}}\left\{\min _{1 \leq i \leq n}\left(\mu_{\tilde{a}_{i}}\left(s_{i}\right) \mid f_{j}\left(s_{1}, \ldots, s_{n}\right) \leq b, j=1, \ldots, m\right\}\right.
$$

Com base nestes conceitos da teoria de possibilidades, os modelos fuzzy DEA (FDEA) são transformados em modelos de possibilidades DEA (PDEA). Esta abordagem permite que o problema possa ser resolvido considerando-se diferentes níveis de possibilidades para o atendimento das restrições e diferentes níveis de possibilidade para o atendimento da função objetivo. Com isto propagam-se as incertezas fuzzy para o resultado. Como solução tem-se um valor para a função objetivo para cada um dos níveis de possibilidades considerados para o atendimento das restrições e para a função objetivo.

\subsection{Modelo de possibilidades DEA}

O conceito de programação restringida por chances ("Chance Constrained Programming" CCP), introduzido por Charnes, Cooper \& Rhodes (1978) é utilizado como um método para solucionar os modelos fuzzy CCR. A resolução via CCP trata as incertezas especificando o nível de confiança com o qual se pode tratar as restrições. Utilizando-se o conceito de CCP e de possibilidade dos eventos fuzzy, o modelo FCCR torna-se o seguinte modelo de possibilidades CCR (PCCR):

$\operatorname{Max} \tilde{f}$

sujeito a:

$\pi\left(\underline{\mathrm{v}}^{\mathrm{T}} \underline{\tilde{y}}_{0} \geq \tilde{f}\right) \geq \beta$

$\pi\left(\underline{\mathrm{u}}^{\mathrm{T}} \underline{\tilde{x}}_{0}=1\right) \geq \alpha_{0}$

$\pi\left(\underline{\mathrm{v}}^{\mathrm{T}} \underline{\tilde{y}}_{0}-\underline{\mathrm{u}}^{\mathrm{T}} \underline{\tilde{x}}_{0} \leq \underline{0}\right) \geq \underline{\alpha}$

$\underline{u}, \underline{v} \geq \underline{0}$ 
onde $\beta$ e $\alpha_{0} \in[0,1]$ são os níveis de possibilidade aceitáveis pré-estabelecidos para a primeira e a segunda restrições, respectivamente, da formulação (17). $\underline{\alpha}=\left[\alpha_{1}, \alpha_{2}, \ldots, \alpha_{n}\right]^{T} \in[0,1]^{n}$ é um vetor coluna de níveis de possibilidades aceitáveis para o vetor de possibilidades da terceira restrição, e $\pi\left(\tilde{f} \leq \underline{v}^{T} \tilde{y}_{0}\right)=\sup _{s, t \in \Re}\left\{\min \left(\mu_{\tilde{f}}(s), \underline{\mu}_{\underline{v}^{T} \tilde{y}_{0}}(t)\right) \mid s \leq t\right\}$, conforme definido em (Lertworasirikul et al., 2003).

A formulação (17) preconiza que a solução ótima é obtida quando se alcança valores para $\underline{v}^{T} \tilde{y}_{0}$ pelo menos iguais ao valor de $\tilde{f}$ com nível de possibilidade $\beta$, enquanto que, ao mesmo tempo, todas as restrições são satisfeitas para um nível de possibilidade préestabelecido.

Definição 1. Uma DMU é dita $\alpha$-possibilística eficiente se $\tilde{f}$ for maior ou igual do que 1 para este dado nível de possibilidade $\alpha$.

Definição 2. Dada uma variável fuzzy $\tilde{a}$ num espaço de possibilidades $(\Theta, \wp(\Theta), \pi)$, a variável fuzzy $\tilde{a}$ é normal se $\sup _{s \in \Re} \mu_{\tilde{a}}(s)=1$.

Definição 3. Um conjunto de nível $\alpha$ de uma variável fuzzy $\tilde{a}$ é definido por um conjunto de elementos que pertencem à variável $\tilde{a}$ com grau de pertinência de pelo menos $\alpha$, isto é, $a_{\alpha}=\left\{s \in \mathfrak{R} \mid \mu_{\tilde{a}}(s) \geq \alpha\right\}$.

Definição 4. Uma variável fuzzy ã é convexa se:

$$
\mu_{\tilde{a}}\left(\lambda s_{1}+(1-\lambda) s_{2}\right) \geq \min \left(\mu_{\tilde{a}}\left(s_{1}\right), \mu_{\tilde{a}}\left(s_{2}\right)\right) \quad \forall \mathrm{s}_{1}, \mathrm{~s}_{2} \in \mathfrak{R}, \lambda \in[0,1] .
$$

De forma alternativa, uma variável fuzzy $\tilde{a}$ é convexa se ela for convexa para todos os níveis $\alpha$ de pertinência.

Lema 1. Sejam $\tilde{a}_{1}, \tilde{a}_{2}, \ldots, \tilde{a}_{n}$ variáveis fuzzy com função de pertinência normais e convexas. Seja, também, $(\bullet)_{\alpha_{i}}^{\mathrm{L}}$ e $(\bullet)_{\alpha_{\mathrm{i}}}^{\mathrm{U}}$ os limites inferior e superior do conjunto de possibilidade $\alpha$ de $\tilde{a}_{\mathrm{i}}, \mathrm{i}=1, \ldots, \mathrm{n}$. Diante disto, para quaisquer níveis de possibilidades $\alpha_{1}, \alpha_{2}$ e $\alpha_{3}$, com $0 \leq \alpha_{1}$, $\alpha_{2}, \alpha_{3} \leq 1$, as seguintes afirmações são verdadeiras:

$$
\begin{aligned}
& \text { i. } \pi\left(\sum_{\mathrm{i}=1}^{\mathrm{n}} \tilde{\mathrm{a}}_{\mathrm{i}} \leq \mathrm{b}\right) \geq \alpha_{1} \text { se, e somente se, } \sum_{\mathrm{i}=1}^{\mathrm{n}}\left(\tilde{\mathrm{a}}_{\mathrm{i}}\right)_{\alpha_{1}}^{\mathrm{L}} \leq \mathrm{b} ; \\
& \text { ii. } \pi\left(\sum_{\mathrm{i}=1}^{\mathrm{n}} \tilde{\mathrm{a}}_{\mathrm{i}} \geq \mathrm{b}\right) \geq \alpha_{2} \text { se, e somente se, } \sum_{\mathrm{i}=1}^{\mathrm{n}}\left(\tilde{\mathrm{a}}_{\mathrm{i}}\right)_{\alpha_{2}}^{\mathrm{U}} \geq \mathrm{b} ; \\
& \text { iii. } \pi\left(\sum \tilde{\mathrm{a}}_{\mathrm{i}}=\mathrm{b}\right) \geq \alpha_{3} \text { se, e somente se, } \sum_{\mathrm{i}=1}^{\mathrm{n}}\left(\tilde{\mathrm{a}}_{\mathrm{i}}\right)_{\alpha_{3}}^{\mathrm{L}} \leq \mathrm{b} \text { e } \sum_{\mathrm{i}=1}^{\mathrm{n}}\left(\tilde{\mathrm{a}}_{\mathrm{i}}\right)_{\alpha_{3}}^{\mathrm{U}} \geq \mathrm{b} .
\end{aligned}
$$

Dado que os conjuntos fuzzy de entrada e saída para o modelo PCCR são normais e convexos, segue do Lema 1 que o modelo PCCR pode ser resolvido considerando-o da seguinte forma: 
$\operatorname{Max} \tilde{\mathrm{f}}$

sujeito a:

$\left(\underline{v}^{\mathrm{T}} \underline{\mathrm{y}}_{0}\right)_{\beta}^{\mathrm{U}} \geq \tilde{\mathrm{f}}$

$\left(\underline{u}^{\mathrm{T}} \tilde{\mathrm{x}}_{0}\right)_{\alpha_{0}}^{\mathrm{U}} \geq 1$

$\left(\underline{\mathrm{u}}^{\mathrm{T}} \underline{\mathrm{x}}_{0}\right)_{\alpha_{0}}^{\mathrm{L}} \leq 1$

$\left(\underline{\mathrm{v}}^{\mathrm{T}} \underline{\tilde{y}}_{0}-\underline{\mathrm{u}}^{\mathrm{T}} \underline{\tilde{x}}_{0}\right)_{\alpha}^{\mathrm{L}} \leq \underline{0}$

$\underline{\mathrm{u}}, \underline{\mathrm{v}} \geq \underline{0}$

Dependendo da forma da função de pertinência, o modelo supracitado pode ser linear ou não.

Para os casos em que as funções de pertinência sejam trapezoidais, conforme a Figura 3, o modelo pode ser formulado como se segue:

$\operatorname{Max}_{\mathrm{u}, \mathrm{v}, \overline{\mathrm{f}}} \tilde{\mathrm{f}}$

sujeito a :

$$
\begin{aligned}
&(1-\beta)\left(\mathrm{v}^{\mathrm{t}} \tilde{y}_{0}\right)_{0}^{\mathrm{U}}+\beta\left(\mathrm{v}^{\mathrm{t}} \tilde{\mathrm{y}}_{0}\right)_{1}^{\mathrm{U}} \geq \tilde{\mathrm{f}} \\
&\left(1-\alpha_{0}\right)\left(\mathrm{u}^{\mathrm{t}} \tilde{\mathrm{x}}_{0}\right)_{0}^{\mathrm{U}}+\alpha_{0}\left(\mathrm{u}^{\mathrm{t}} \tilde{\mathrm{x}}_{0}\right)_{1}^{\mathrm{U}} \geq 1 \\
&\left(1-\alpha_{0}\right)\left(\mathrm{u}^{\mathrm{t}} \tilde{\mathrm{x}}_{0}\right)_{0}^{\mathrm{U}}+\alpha_{0}\left(\mathrm{u}^{\mathrm{t}} \tilde{\mathrm{x}}_{0}\right)_{1}^{\mathrm{U}} \leq 1 \\
&(1-\mathrm{A})\left(\left(-\underline{\mathrm{u}}^{\mathrm{t}} \underline{\underline{\mathrm{X}}}\right)_{0}^{\mathrm{L}}+\left(\underline{\mathrm{v}}^{\mathrm{t}} \tilde{\underline{Y}}\right)_{0}^{\mathrm{L}}\right)+\mathrm{A}\left(\left(-\underline{\mathrm{u}}^{\mathrm{t}} \tilde{\underline{X}}\right)_{1}^{\mathrm{L}}+\left(\underline{\mathrm{v}}^{\mathrm{t}} \underline{\underline{Y}}\right)_{1}^{\mathrm{L}}\right) \leq \underline{0} \\
& \mathrm{u}, \mathrm{v} \geq 0
\end{aligned}
$$

É importante notar que as funções triangulares são casos particulares das trapezoidais, onde os limites inferiores e superiores para $\alpha=1$ são iguais.

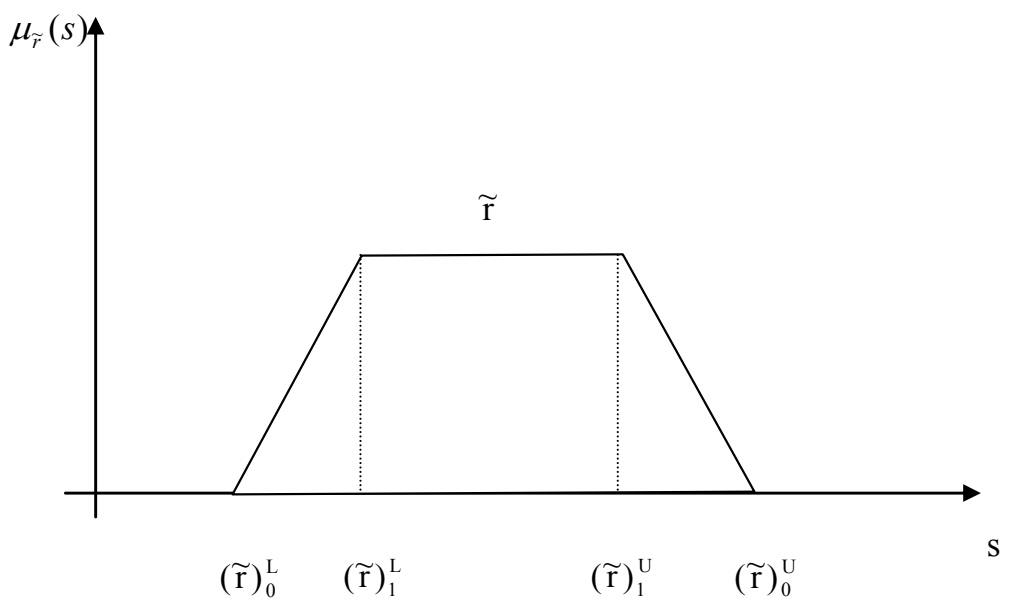

Figura 3 - Número fuzzy trapezoidal. 


\subsection{Adaptação do modelo de possibilidades DEA para a análise de modos de falha}

Conforme já mencionado, o que se pretende é utilizar a essência da modelagem apresentada na seção anterior para priorizar modos de falhas levantados na FMEA. Para esta priorização considerar-se-ão os índices de ocorrência, gravidade e detecção de modo a estabelecer-se um grau de criticidade relativa entre os modos de falha.

Tomado por base as considerações feitas em Garcia (2001), Garcia et al. (2002) e Lertworasirikul (2001) e também, por questões de facilidade nos cálculos, o fato de se estar trabalhando com números fuzzy triangulares faz com que a formulação (19) possa ser modelada da seguinte maneira:

$$
\begin{aligned}
& \operatorname{Max}_{\mathrm{u}, \mathrm{v}, \mathrm{f}} \tilde{\mathrm{f}} \\
& \text { sujeito a : } \\
& (1-\alpha)\left(\mathrm{v}^{\mathrm{t}} \tilde{\mathrm{y}}_{0}\right)_{0}^{\mathrm{U}}+\alpha\left(\mathrm{v}^{\mathrm{t}} \tilde{\mathrm{y}}_{0}\right)_{1}^{\mathrm{U}} \geq \tilde{\mathrm{f}} \\
& (1-\alpha)\left((-1)_{0}^{\mathrm{L}}+\left(\underline{\mathrm{v}}^{\mathrm{t}} \underline{\tilde{Y}}\right)_{0}^{\mathrm{L}}\right)+\alpha\left((-1)_{1}^{\mathrm{L}}+\left(\underline{\mathrm{v}}^{\mathrm{t}} \underline{\tilde{Y}}\right)_{1}^{\mathrm{L}}\right) \leq \underline{0} \\
& \mathrm{u}, \mathrm{v} \geq 0
\end{aligned}
$$

Observe-se que nesta modelagem a segunda e a terceira restrições da formulação (20) desaparecem, tendo em vista que se está assumindo um valor real, igual e constante para todos os modos de falha.

Uma constatação feita por Garcia (2001) está relacionada com o poder discriminatório para o caso da priorização dos modos de falha. Um dos problemas associados à utilização do tradicional número de priorização dos riscos, comumente adotado nos padrões de FMEA (US Army, 1980; AIAG, 1995), refere-se à importância dos índices utilizados.

Diante desta constatação, Garcia (2001 e 2002) adota uma modelagem denominada "Agregação Parcial das Eficiências com Gravidade Fixa", ou seja, são obtidos os graus de eficiências parciais combinando-se primeiramente os índices de ocorrência e de gravidade, e posteriormente combinando-se os índices de gravidade e detecção. A medida de criticidade relativa final é obtida calculando a média das eficiências parciais. Neste trabalho utiliza-se a mesma abordagem, porém utilizando-se índices possibilísticos. Desta forma o modelo utilizado é denominado de modelo de "Possibilidades com Agregação Parcial das Eficiências com Gravidade Fixa" - PAPGF.

\section{Aplicação do modelo PAPGF para priorizar os modos de falha do CVCS}

Inicialmente, são reunidos os especialistas envolvidos na análise para que os mesmos determinem as funções de pertinência para as variáveis linguísticas que serão adotadas. As variáveis a serem adotadas, assim como as suas interpretações, podem ser vistas na Tabela 1. 
Tabela 1 - Definição das variáveis linguísticas utilizadas pelos especialistas.

\begin{tabular}{|c|c|c|c|}
\hline $\begin{array}{l}\text { Variáveis } \\
\text { linguísticas }\end{array}$ & Gravidade & Ocorrência & Detecção \\
\hline Muito alta & $\begin{array}{l}\text { O efeito da falha pode afetar tanto } \\
\text { a segurança operacional como a } \\
\text { ambiental, podendo ocasionar } \\
\text { danos a bens ou pessoas e/ou } \\
\text { infringir alguma legislação ou } \\
\text { regulamentação governamental. }\end{array}$ & $\begin{array}{l}\text { Praticamente } \\
\text { inevitável }\end{array}$ & $\begin{array}{l}\text { A falha permanece oculta } \\
\text { até que o desempenho do } \\
\text { sistema se degrade até que } \\
\text { o mesmo não consiga } \\
\text { cumprir sua missão }\end{array}$ \\
\hline Alta & $\begin{array}{l}\text { Afeta a eficiência do sistema ao } \\
\text { qual está associado e/ou da } \\
\text { planta, ocasionando uma redução } \\
\text { de potência ou até mesmo o seu } \\
\text { desligamento. }\end{array}$ & Frequente & $\begin{array}{l}\text { A falha permanece oculta } \\
\text { até que o desempenho do } \\
\text { sistema se reduza } \\
\text { consideravelmente }\end{array}$ \\
\hline Moderada & $\begin{array}{l}\text { Reduz a eficiência do sistema ao } \\
\text { qual está associado, gerando um } \\
\text { estresse no ambiente de trabalho } \\
\text { que leva a planta a operar num } \\
\text { nível de risco mais elevado do } \\
\text { que o normal. }\end{array}$ & Ocasional & $\begin{array}{c}\text { A falha permanece } \\
\text { despercebida até que o } \\
\text { desempenho do sistema seja } \\
\text { reduzido }\end{array}$ \\
\hline Baixa & $\begin{array}{l}\text { O efeito da falha não interfere na } \\
\text { operação da planta, reduzindo de } \\
\text { forma mínima somente o desem- } \\
\text { penho do sistema. }\end{array}$ & Poucas vezes & $\begin{array}{l}\text { A falha permanece oculta } \\
\text { até que seja realizada uma } \\
\text { inspeção ou um teste }\end{array}$ \\
\hline Remota & $\begin{array}{l}\text { O efeito da falha praticamente } \\
\text { não é percebido. }\end{array}$ & $\begin{array}{l}\text { Excepcional- } \\
\text { mente }\end{array}$ & $\begin{array}{l}\text { A falha permanece oculta; e } \\
\text { o defeito será prontamente } \\
\text { percebido quando de um } \\
\text { teste ou de uma inspeção }\end{array}$ \\
\hline
\end{tabular}

Nota: O índice de gravidade está relacionado com os efeitos da ocorrência do modo de falha sob análise, o índice de ocorrência está relacionado com a frequência (ou probabilidade) de ocorrência do modo de falha, e o índice de detecção está relacionado com o potencial de identificação do problema antes que as consequências ocorram.

A amplitude de variação para cada variável foi estabelecida considerando-se a opinião de cinco especialistas, conforme pode ser visto na Tabela 2.

Nessa tabela tem-se o valor $\beta$, que representa o peso associado ao especialista, a, b e c são os vértices da função de pertinência fornecida pelos especialistas, de modo que $\alpha$ é o grau de pertinência do elemento do domínio na função. Ou seja, para $\alpha=0$ têm-se os vértices da base do triângulo ou trapézio, enquanto que para $\alpha=1$ têm-se os vértices opostos à base do triângulo, ou à base maior do trapézio caso esta esteja apoiada sobre o eixo das abscissas.

Note-se que com isto, na última linha (total) têm-se os valores característicos finais considerando-se uma média ponderada das opiniões dos especialistas. Por exemplo, considerando a coluna referente ao nível baixo (B) para $\alpha=0$ tem-se: $0,3 \times 2+0,2 \times 1,5+0,2 \times 1,8+0,2 \times 1,9+0,1 \times 1,7=1,81$. Este valor corresponde ao vértice a da função de pertinência triangular associada à variável linguística "baixo". O mesmo raciocínio se aplica às demais variáveis linguísticas. 
Tabela 2 - Caracterização dos níveis das variáveis linguísticas utilizadas pelos especialistas.

\begin{tabular}{|l|l|l|l|l|l|l|l|l|l|l|l|l|l|l|l|l|}
\hline Especialista & $\boldsymbol{\beta}$ & \multicolumn{9}{|c|}{$\mathbf{a}(\boldsymbol{\alpha}=\mathbf{0})$} & \multicolumn{4}{|c|}{$\mathbf{b}(\boldsymbol{\alpha}=\mathbf{1})$} & \multicolumn{5}{c|}{$\mathbf{c}(\boldsymbol{\alpha}=\mathbf{0})$} \\
\hline & & $\mathbf{R}^{*}$ & $\mathbf{B}$ & $\mathbf{M}$ & $\mathbf{A}$ & $\mathbf{M A}$ & $\mathbf{R}$ & $\mathbf{B}$ & $\mathbf{M}$ & $\mathbf{A}$ & $\mathbf{M A}$ & $\mathbf{R}$ & $\mathbf{B}$ & $\mathbf{M}$ & $\mathbf{A}$ & $\mathbf{M A}$ \\
\hline $\mathbf{1}$ & 0.3 & 0 & 2 & 4 & 7 & 9 & 1.5 & 3.5 & 6 & 8.5 & 10 & 3 & 5 & 8 & 10 & 10 \\
\hline $\mathbf{2}$ & 0.2 & 0 & 1.5 & 3.5 & 6 & 8.5 & 1.7 & 3.2 & 5.5 & 8.0 & 10 & 2.8 & 4.8 & 7.8 & 10 & 10 \\
\hline $\mathbf{3}$ & 0.2 & 0 & 1.8 & 3.7 & 6.8 & 8.7 & 1.6 & 3.7 & 5.7 & 8.8 & 10 & 2.5 & 5.3 & 8.4 & 10 & 10 \\
\hline $\mathbf{4}$ & 0.2 & 0 & 1.9 & 3.8 & 7.2 & 9.4 & 1.4 & 3.6 & 5.8 & 8.7 & 10 & 3.2 & 5.3 & 8.2 & 10 & 10 \\
\hline $\mathbf{5}$ & 0.1 & 0 & 1.7 & 3.6 & 6.9 & 8.8 & 1.5 & 3.5 & 5.8 & 8.1 & 10 & 2.9 & 5.0 & 8.2 & 10 & 10 \\
\hline Total & 1 & 0 & 1.8 & 4.1 & 6.8 & 8.9 & 1.5 & 3.5 & 5.8 & 8.4 & 10 & 2.9 & 5.0 & 8.1 & 10 & 10 \\
\hline
\end{tabular}

* Para o Remoto (R), deve-se considerá-lo como sendo um número fuzzy trapezoidal. Sendo assim, onde se lê $\alpha=0$ deve-se entender como sendo $\alpha=1$, somente para este caso, conforme pode ser visto na Figura 5 . As abreviaturas são: R - Remoto, B - Baixo, M - Médio, A - Alto e MA - Muito Alto.

As representações gráficas das funções de pertinência, associadas a cada variável linguística definida na Tabela 2, caracterizadas pela linha total, podem ser vistas na Figura 4.

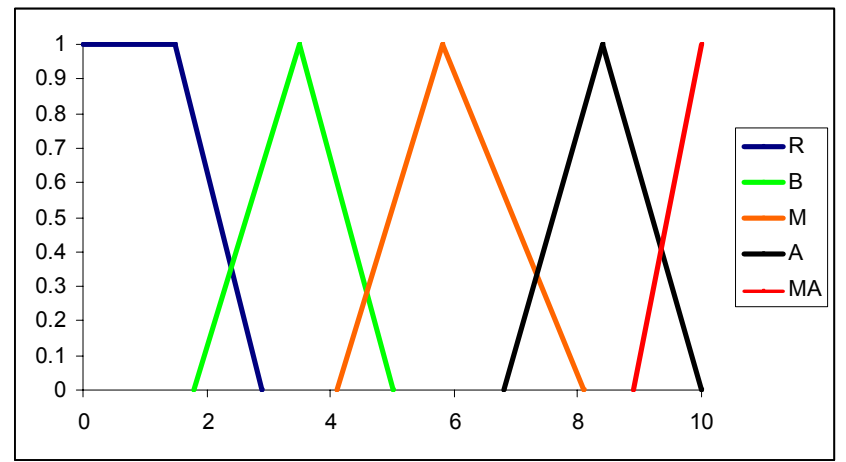

Figura 4 - Representação gráfica dos níveis das variáveis linguísticas utilizadas pelos especialistas.

Estabelecidas as funções de pertinência para cada variável, pode-se então realizar a análise do sistema por meio da FMEA, sendo que para cada índice da análise (ocorrência, gravidade e detecção), são associadas as respectivas variáveis linguísticas, de acordo com a opinião dos especialistas. As planilhas da FMEA para o CVCS podem ser vistas em Lapa \& Guimarães (2004).

\section{Resultados}

Note-se que, como se está trabalhando com cinco níveis linguísticos para cada índice, e foram identificados oitenta e dois modos de falha, são grandes as chances de se terem combinações iguais para modos de falha diferentes. O Anexo 1 apresenta os níveis atribuídos a todos os oitenta e dois modos de falha identificados.. Devido a isto, os modos de falha pertencentes à mesma classe terão criticidades relativas iguais. Para o caso do CVCS, foram destacados, dentre os oitenta e dois modos de falha identificados, 19 classes distintas, conforme mostrado na Tabela 3. 
Tabela 3 - Classes com as mesmas características.

\begin{tabular}{|c|c|c|c|}
\hline Classes & Ocorrência & Gravidade & Detecção \\
\hline $\mathbf{1}$ & $\mathrm{B}$ & $\mathrm{A}$ & $\mathrm{A}$ \\
\hline $\mathbf{2}$ & $\mathrm{B}$ & $\mathrm{A}$ & $\mathrm{M}$ \\
\hline $\mathbf{3}$ & $\mathrm{B}$ & $\mathrm{B}$ & $\mathrm{M}$ \\
\hline $\mathbf{4}$ & $\mathrm{B}$ & $\mathrm{M}$ & $\mathrm{B}$ \\
\hline $\mathbf{5}$ & $\mathrm{B}$ & $\mathrm{M}$ & $\mathrm{M}$ \\
\hline $\mathbf{6}$ & $\mathrm{B}$ & $\mathrm{MA}$ & $\mathrm{M}$ \\
\hline $\mathbf{7}$ & $\mathrm{B}$ & $\mathrm{R}$ & $\mathrm{M}$ \\
\hline $\mathbf{8}$ & $\mathrm{M}$ & $\mathrm{A}$ & $\mathrm{A}$ \\
\hline $\mathbf{9}$ & $\mathrm{M}$ & $\mathrm{A}$ & $\mathrm{M}$ \\
\hline $\mathbf{1 0}$ & $\mathrm{M}$ & $\mathrm{B}$ & $\mathrm{M}$ \\
\hline $\mathbf{1 1}$ & $\mathrm{M}$ & $\mathrm{M}$ & $\mathrm{B}$ \\
\hline $\mathbf{1 2}$ & $\mathrm{M}$ & $\mathrm{M}$ & $\mathrm{M}$ \\
\hline 13 & $\mathrm{M}$ & $\mathrm{MA}$ & $\mathrm{B}$ \\
\hline 14 & $\mathrm{M}$ & $\mathrm{MA}$ & $\mathrm{M}$ \\
\hline $\mathbf{1 5}$ & $\mathrm{M}$ & $\mathrm{MA}$ & $\mathrm{MA}$ \\
\hline $\mathbf{1 6}$ & $\mathrm{M}$ & $\mathrm{R}$ & $\mathrm{M}$ \\
\hline $\mathbf{1 7}$ & $\mathrm{R}$ & $\mathrm{A}$ & $\mathrm{M}$ \\
\hline $\mathbf{1 8}$ & $\mathrm{R}$ & $\mathrm{M}$ & $\mathrm{B}$ \\
\hline $\mathbf{1 9}$ & $\mathrm{R}$ & $\mathrm{M}$ & $\mathrm{M}$ \\
\hline
\end{tabular}

Na Tabela 4 são apresentados os resultados obtidos, para cada uma das 19 classes, pela modelagem proposta neste artigo.

Tabela 4 - Priorização dos modos de falha por meio do modelo PAPGF.

\begin{tabular}{|c|c|c|c|c|c|}
\hline Classes & ${ }^{* *}$ PAPGF & ${ }^{*} \mathbf{R}_{\text {PAPGF }}$ & Classes & PAPGF & R PAPGF \\
\hline $\mathbf{1}$ & 0,982773 & 3 & $\mathbf{1 1}$ & 0,809864 & 7 \\
\hline $\mathbf{2}$ & 0,982773 & 3 & $\mathbf{1 2}$ & 0,809864 & 7 \\
\hline $\mathbf{3}$ & 0,810773 & 6 & $\mathbf{1 3}$ & 1,065091 & 1 \\
\hline $\mathbf{4}$ & 0,811682 & 5 & $\mathbf{1 4}$ & 1,065091 & 1 \\
\hline $\mathbf{5}$ & 0,811682 & 5 & $\mathbf{1 5}$ & 1,065091 & 1 \\
\hline $\mathbf{6}$ & 1,065091 & 1 & $\mathbf{1 6}$ & 0,809864 & 7 \\
\hline $\mathbf{7}$ & 0,809864 & 7 & $\mathbf{1 7}$ & 0,975955 & 4 \\
\hline $\mathbf{8}$ & 0,983227 & 2 & $\mathbf{1 8}$ & 0,7385 & 8 \\
\hline $\mathbf{9}$ & 0,983227 & 2 & $\mathbf{1 9}$ & 0,7385 & 8 \\
\hline $\mathbf{1 0}$ & 0,809864 & 7 & - & - & - \\
\hline
\end{tabular}

${ }^{*} \mathrm{R}_{\mathrm{PAPGF}}$ é a priorização do grupo segundo o modelo PAPGF

***APGF é a criticidade relativa do grupo de modos de falha 
$\mathrm{Na}$ Tabela 4, as colunas com cabeçalho PAPGF dizem respeito às criticidades relativas associadas aos grupos de modos de falha, enquanto que as colunas com cabeçalho $\mathbf{R}_{\mathbf{P A P G F}}$ dizem respeito à priorização dada ao grupo, baseando-se na criticidade relativa a ele associada.

Uma aplicação de sistemas de inferência fuzzy para priorizar os modos de falha do CVCS foi apresentada em Lapa \& Guimarães (2004). Nesse artigo os autores utilizaram um sistema de inferência fuzzy considerando cinco níveis para os índices de ocorrência, gravidade e detecção respectivamente. Estes índices foram combinados por meio de quatorze regras de inferência sobre uma variável de saída para o risco. Essa variável de saída foi subdividida em seis níveis. Mais detalhes podem ser vistos na referência supracitada.

Os resultados obtidos em Lapa \& Guimarães (2004) coincidiram parcialmente com os obtidos no presente trabalho, o que era de se esperar. Acredita-se que as diferenças deram-se pelo fato de, na abordagem proposta pelos referidos autores, serem utilizadas estimativas pontuais "crisp" para os índices e esses são utilizados com fatores de entrada. Na priorização feita pelo modelo APGF não são utilizadas as estimativas "crisp" como dados de entrada, mas sim os níveis dos índices definidos pelas respectivas funções de pertinência, ou seja, possibilidades. Com esta diferença na abordagem proposta os autores acreditam ser um diferencial operacional, tendo em vista que os especialistas não precisam estabelecer regras de inferência, em virtude do que já é preconizado pelos manuais de referência Mil-std 1629 (US Army, 1980) e QS9000 (AIAG, 1995). No entanto, essas diferenças não podem ser estritamente julgadas tendo em vista que, em se tratando de uma abordagem fuzzy, os índices são dependentes das interpretações dos especialistas e, consequentemente, os resultados são influenciados pelas características dos índices.

Cabe ressaltar, no entanto, que o objetivo do presente trabalho não é comparar os resultados obtidos pelas diversas abordagens, mas sim apresentar um método que, do ponto de execução apresente vantagens em relação aos já apresentados e, sobretudo, evite os problemas apresentados por Bowles \& Bonnell (1998) e Bowles (1998 e 2003), associados ao número de priorização de riscos (RPN - "Risk Priority Number") tradicional. Os benefícios associados ao método proposto em relação ao RPN podem ser visto em Garcia et al. (2005a) e Garcia (2006), quando os autores aplicam esta abordagem para o sistema auxiliar de água de alimentação de uma usina nuclear. No entanto, nessa aplicação o número de modos de falha considerados é substancialmente inferior aos considerados na presente aplicação.

O resultado obtido com a abordagem proposta foi considerado adequado e de grande relevância pela equipe de especialistas envolvida na análise. De acordo com a experiência operacional dos especialistas envolvidos, esta priorização reflete o que vem sendo praticado há aproximadamente quinze anos. Tendo em vista este fato, acredita-se que, para sistemas mais novos, onde não se tem uma gama de informação como para o caso do CVCS, os sentimentos e percepções dos especialistas, refletidos nas funções de pertinência, conduzam a uma priorização conveniente dos modos de falha.

É importante destacar que a obtenção dos resultados apresentados na Tabela 4 se deu de forma totalmente diferente de como é preconizado pelas diferentes abordagens fundamentadas em lógica fuzzy (Lapa \& Guimarães, 2004; Peláez \& Bowles, 1996; Xu et al., 2002). A principal característica da abordagem proposta está no fato de não ser necessário, durante o desenvolvimento da FMEA, que se extraia dos especialistas um valor numérico numa escala de 1 a 10 para associar aos índices de ocorrência, gravidade e detecção, respectivamente. Para a priorização dos modos de falha, basta que os técnicos forneçam por meio das 
expressões linguísticas o nível dos índices como sendo remoto, baixo, moderado, alto ou muito alto.

Do ponto de vista de discriminação, a abordagem calcada em inferência fuzzy, baseada em um banco de regras especialistas, separou os 82 modos de falha do sistema em 8 grupos, da mesma forma que a abordagem aqui proposta. As classificações ficaram muito próximas umas das outras. As diferenças estão relacionadas com as regras utilizadas, que dependerão do grupo de especialistas. Tal fato não ocorre com a abordagem apresentada por Lapa \& Guimarães (2004), tendo em vista que o modelo será sempre o mesmo, as distinções estarão somente no que se refere ao mapeamento das variáveis linguísticas e no número de níveis a serem considerados. Nesse trabalho, os autores utilizaram 4 variáveis, 3 de entrada, relacionadas com os índices de ocorrência, gravidade e detecção, e uma quarta variável associada ao risco. Esta última variável foi classificada em 6 níveis.

Note-se que, na abordagem proposta no presente trabalho, não é necessária a inserção de uma variável para caracterizar uma resposta. O resultado da priorização é obtido a partir das próprias variáveis do problema inicial. Este fato traz uma vantagem de cunho prático, tendo em vista não ser necessária a criação de uma nova variável e nem ser necessário possuir três valores reais de entrada para serem submetidos ao banco de regras especialistas.

\section{Conclusões}

Quando se realiza uma FMEA para propósitos de segurança, não se deve ater a grandes precisões dos dados, principalmente quando se está analisando um sistema em sua fase de projeto, ou seja, em fase conceitual. Para análises como estas, recomenda-se que se adquiram informações de especialistas.

O uso de variáveis linguísticas, provinda de especialistas, fornece informações significativas com respeito ao sistema e aos índices utilizados para classificar os modos de falha. Para tais variáveis, a consideração das incertezas associadas é fator importante, pois reflete o grau de conhecimento sobre o sistema em análise.

As vantagens observadas pela utilização de um modelo híbrido, combinando os conceitos de lógica fuzzy e análise envoltória de dados, podem ser sintetizadas da seguinte forma:

- Pode ser aplicado na fase inicial de projeto, em que os dados disponíveis são escassos e imprecisos;

- É uma técnica que combina, de forma organizada, o conhecimento dos especialistas, assim como as incertezas associadas aos mesmos;

- A flexibilidade em se definir os pesos associados com cada fator provê uma maneira de se verificar, por meio do modelo DEA, quais índices estão contribuindo de forma mais efetiva para a sua criticidade;

- Com a utilização do modelo PAPGF torna-se desnecessário o estabelecimento de inferências que levem a uma combinação de inputs num universo restrito de conjuntos de saída;

- Não é necessário que se tenha um índice específico para que se possa inferir sobre o grau de criticidade dos modos de falha, como é o caso da inferência fuzzy tradicional;

- Os resultados obtidos pela abordagem proposta são relativos, ou seja, é uma abordagem onde os resultados são dependentes do conjunto de modos de falha em análise. 
Um fator importante associado com a abordagem proposta é que os modos de falha com características diferentes não são priorizados da mesma forma. Somente modos de falha em que o índice de gravidade é relativamente elevado e modos de falha que possuem uma combinação dos índices de ocorrência e detecção, relativamente elevados, são considerados $100 \%$ críticos. Mais detalhes sobre esta observação podem ser vistos em Garcia (2001 e 2006) e Garcia et al. (2005a).

Tendo em vista a potencialidade observada em se utilizar modelos baseados em DEA para comparar os riscos entre diferentes entidades (modos de falha, tecnologias etc.) sugere-se que seja estudada a utilização desta abordagem para se identificar fatores de melhoria em processos. Tendo em vista que os modelos DEA estabelecem um conjunto de pesos que maximizam a eficiência ou, dependendo da ótica, a ineficiência, como foi o caso aplicado neste trabalho, sugere-se também que seja estabelecida uma abordagem onde se possam identificar os pontos eficientes e, a partir destes, estabelecer melhorias no sistema em análise (Garcia et al., 2005b).

\section{Agradecimentos}

Os autores gostariam de agradecer enormemente a colaboração dos revisores anônimos para que o trabalho alcançasse o nível apresentado.

\section{Referências Bibliográficas}

(1) Ayyub, B.M. (2001). Elicitation of Expert Opinions for Uncertainty and Risks. CRC Press LLC, Boca Raton, Florida.

(2) Bowles, J.B. \& Peláez, C.E. (1995). Fuzzy Logic Prioritization of Failures in System Failure Mode, Effect and Criticality Analysis. Reliability Engineering and System Safety, 50, 203-213.

(3) Bowles, J.B. \& Bonnell, R.D. (1998). Failure Mode Effects and Criticality Analysis: What it is and How to use it. In: Topics in Reliability \& Maintainability \& Statistics, Annual Reliability and Maintainability Symposium, Anaheim, CA, January.

(4) Bowles, J.B. (1998). The new SAE FMECA standard. In: IEEE Proceedings Annual Reliability and Maintainability Symposium, 48-53.

(5) Bowles, J.B. (2003). An assessment of RPN prioritization in a failure modes effects and criticality analysis. In: IEEE Proceedings annual Reliability and Maintainability Symposium, 380-386.

(6) Charnes, A.; Cooper, W.W. \& Rhodes, E. (1978). Measuring The Efficiency of Decision Making Units. European Journal of Operational Research, 2, 429-444.

(7) Chrysler Corporation; Ford Motor Company and General Motors (1994). Potential Failure Mode and Effect Analysis, FMEA, Third Edition. Quality System Requirement QS-9000.

(8) De Souza, D.P.H. (2003). Avaliação de métodos paramétricos e não paramétricos na análise de eficiência da produção de leite. Tese de D.Sc., Escola Superior de Agricultura, Universidade de São Paulo, Piracicaba - SP. 
(9) EPRI (1996). Risk informed inservice inspection evaluation procedure. Electric Power Research Institur, TR-106706.

(10) Ekel, P.Y. \& Schuffner Neto, F.H. (2006). Algorithms of discrete optimization and their application to problems with fuzzy coefficients. Information Sciences, 176, 2846-2868.

(11) Farrel, M.J. (1957). The Measurement of Productive Efficiency. Journal of the Royal Statistical Society, Series A, part III, 253-290.

(12) Galperin, E.A. \& Ekel, P.Y. (2005). Synthetic realization approach to fuzzy global optimization via gamma algorithm. Mathematical and Computer Modelling, 41, 1457-1468.

(13) Garcia, P.A.A. (2006). Utilização de um modelo fuzzy de envelopamento dos dados da análise dos modos e efeitos de falha. Tese de D.Sc., Programa de Engenharia Nuclear, COPPE/UFRJ, Rio de Janeiro.

(14) Garcia, P.A.A. (2001). Aplicação de análise envoltória de dados no processo de manutenção centrada em confiabilidade. Dissertação de M.Sc., Departamento de Sistemas e Computação, Instituto Militar de Engenharia, Rio de Janeiro.

(15) Garcia, P.A.A.; Neves, J.C.C. \& Neves, G.C. (2002). Avaliação dos modos e efeitos de falha no RCM utilizando DEA. XI Congreso Latino-Iberoamericano de Investigación de Operaciones, Conceição, Chile.

(16) Garcia, P.A.A.; Frutuoso e Melo, P.F.F. \& Schirru, R. (2005a). Fuzzy data envelopment analysis approach for FMEA. Progress in Nuclear Energy, 46, 359-373.

(17) Garcia, P.A.A.; Frutuoso e Melo, P.F.F. \& Varanda Jr., G.B. (2005b). Utilização de um modelo de envelopamento de dados para propor melhorias em segurança: uma abordagem baseada em FMEA. Annals of the International Nuclear Atlantic Conference, Santos-SP, Brasil.

(18) Grove, E.J. \& Travis, R.J. (1995). Effect of aging on the PWR chemical and volumetric control system. NUREG/CR-5954, BNL - NUREG-52410.

(19) Guo, P. \& Tanaka, H. (2001). Fuzzy DEA: a perceptual evaluation method. Fuzzy Sets and Systems, 119, 149-160.

(20) IAEA - International Atomic Energy Agency (2001). Applications of probabilistic safety assessment (PSA) for nuclear power plant. Technical Document 1200, International Atomic Energy Agency, Vienna.

(21) IAEA - International Atomic Energy Agency (2002). Procedure for conducting probabilistic safety assessment for non-reactor nuclear facilities. Technical Document 1267, International Atomic Energy Agency, Vienna.

(22) Kahraman, C. \& Tolga, E. (1998). Data envelopment analysis using fuzzy concept. In: Annals of the $28^{\circ}$ International Symposium on Multiple-Valued Logic, 338-343.

(23) Kao, C. \& Liu, S.T. (2000). Fuzzy efficiency measures in data envelopment analysis. Fuzzy Sets and Systems, 113, 427-437.

(24) Lapa, C.M.F. \& Guimarães, A.C.F. (2004). Fuzzy FMEA applied to PWR chemical and volumetric control system. Progress in Nuclear Energy, 44, 31, 191-213. 
(25) Lertworasirikul, S. (2001). Fuzzy data envelopment analysis for supply chain modeling and analysis. Dissertation Proposal in Industrial Engineering, North Carolina State University.

(26) Lertworasirikul, S.; Fang, S.-C.; Joines, J.A.; Nutt, H.L.W. (2003). Fuzzy data envelopment analysis: a possibility approach. Fuzzy Sets and System, 139, 379-394.

(27) Maeda, Y.; Etani, T. \& Tanaka, H. (1998). Fuzzy DEA with interval efficiency. Annals of the $6^{\text {th }}$ European Congress on Intelligent Techniques and Soft Computing, 2, 1067-1071.

(28) Peláez, E.C. \& Bowles, J.B. (1996). Using fuzzy cognitive maps as a system model for failure modes and effects analysis. Information Science, 88, 177-199.

(29) Pillay, A. \& Wang, J. (2003). Modified failure mode and effect analysis using approximate reasoning. Reliability Engineering and System Safety, 79, 69-85.

(30) US Army (1980). Procedure for performing a failure mode, effect and criticality analysis. Mil-Std 1629 (SHIPS), Department of Defense.

(31) Xu, K.; Tang, L.C.; Xie, M.; Ho, S.L. \& Zhu, M.L. (2002). Fuzzy assessment of FMEA for engine systems. Reliability Engineering and System Safety, 75, 17-29.

(32) Zadeh, L.A. (1987). Fuzzy sets and applications: Selected papers. Wiley, New York.

(33) Zadeh, L.A. (1978). Fuzzy sets as a basis for a theory of possibility. Fuzzy Sets and Systems, 1, 3-28.

ANEXO 1 - Níveis linguísticos associados aos índices dos modos de falha no CVCS.

\begin{tabular}{|c|c|c|c|c|c|c|c|}
\hline Modo de falha & O & G & D & Modo de falha & O & G & D \\
\hline $\mathbf{1}$ & B & M & M & $\mathbf{4 2}$ & M & A & A \\
\hline $\mathbf{2}$ & M & A & A & $\mathbf{4 3}$ & B & A & A \\
\hline $\mathbf{3}$ & M & M & B & $\mathbf{4 4}$ & M & A & A \\
\hline $\mathbf{4}$ & M & M & B & $\mathbf{4 5}$ & B & M & B \\
\hline $\mathbf{5}$ & B & M & M & $\mathbf{4 6}$ & M & M & M \\
\hline $\mathbf{6}$ & B & M & M & $\mathbf{4 7}$ & B & M & M \\
\hline $\mathbf{7}$ & B & A & A & $\mathbf{4 8}$ & M & A & M \\
\hline $\mathbf{8}$ & M & MA & MA & $\mathbf{4 9}$ & M & M & M \\
\hline $\mathbf{9}$ & B & M & B & $\mathbf{5 0}$ & M & M & M \\
\hline 10 & M & M & B & $\mathbf{5 1}$ & B & M & M \\
\hline 11 & B & M & M & $\mathbf{5 2}$ & M & M & M \\
\hline 12 & M & M & M & $\mathbf{5 3}$ & M & M & M \\
\hline 13 & M & MA & B & $\mathbf{5 4}$ & M & M & M \\
\hline 14 & M & MA & B & $\mathbf{5 5}$ & M & M & M \\
\hline 15 & B & A & M & $\mathbf{5 6}$ & M & A & M \\
\hline 16 & B & A & M & $\mathbf{5 7}$ & M & A & M \\
\hline 17 & B & M & M & $\mathbf{5 8}$ & M & A & M \\
\hline
\end{tabular}




\begin{tabular}{|c|c|c|c|c|c|c|c|}
\hline $\mathbf{1 8}$ & M & A & A & $\mathbf{5 9}$ & M & M & M \\
\hline $\mathbf{1 9}$ & B & M & B & $\mathbf{6 0}$ & M & M & M \\
\hline $\mathbf{2 0}$ & B & M & B & $\mathbf{6 1}$ & M & M & M \\
\hline $\mathbf{2 1}$ & B & M & B & $\mathbf{6 2}$ & M & A & M \\
\hline $\mathbf{2 2}$ & R & M & M & $\mathbf{6 3}$ & M & R & M \\
\hline $\mathbf{2 3}$ & R & A & M & $\mathbf{6 4}$ & M & M & M \\
\hline $\mathbf{2 4}$ & B & MA & M & $\mathbf{6 5}$ & M & M & M \\
\hline $\mathbf{2 5}$ & B & B & M & $\mathbf{6 6}$ & M & M & M \\
\hline $\mathbf{2 6}$ & B & B & M & $\mathbf{6 7}$ & M & M & M \\
\hline $\mathbf{2 7}$ & B & MA & M & $\mathbf{6 8}$ & M & M & M \\
\hline $\mathbf{2 8}$ & B & A & A & $\mathbf{6 9}$ & M & M & M \\
\hline $\mathbf{2 9}$ & M & B & M & $\mathbf{7 0}$ & M & M & M \\
\hline $\mathbf{3 0}$ & B & M & M & $\mathbf{7 1}$ & M & M & M \\
\hline $\mathbf{3 1}$ & M & A & M & $\mathbf{7 2}$ & M & M & M \\
\hline $\mathbf{3 2}$ & B & M & M & $\mathbf{7 3}$ & B & M & M \\
\hline $\mathbf{3 3}$ & M & M & M & $\mathbf{7 4}$ & B & R & M \\
\hline $\mathbf{3 4}$ & B & M & M & $\mathbf{7 5}$ & M & M & M \\
\hline $\mathbf{3 5}$ & M & A & M & $\mathbf{7 6}$ & M & M & M \\
\hline $\mathbf{3 6}$ & M & MA & M & $\mathbf{7 7}$ & M & A & M \\
\hline $\mathbf{3 7}$ & B & A & A & $\mathbf{7 8}$ & M & A & M \\
\hline $\mathbf{3 8}$ & R & M & B & $\mathbf{7 9}$ & M & M & M \\
\hline $\mathbf{3 9}$ & B & M & M & $\mathbf{8 0}$ & M & M & M \\
\hline $\mathbf{4 0}$ & B & M & B & $\mathbf{8 1}$ & B & M & M \\
\hline $\mathbf{4 1}$ & B & A & A & $\mathbf{8 2}$ & B & R & M \\
\hline
\end{tabular}

\section{Lista de Símbolos}

CCP Chance Constrained Programming

CCR Charnes, Cooper and Rhodes

CVCS Chemical and Volumetric Control System

DEA Data Envelopment Analysis

DMU Decision Make Unit

FCCR Fuzzy Charnes, Cooper and Rhodes

FDEA Fuzzy Data Envelopment Analysis

FMEA Failure Mode and Effect Analysis

PAPGF Possibility Agregação Parcial com Gravidade Fixa

PCCR Possibility Charnes, Cooper and Rhodes

PDEA Possibility Data Envelopment Analysis

PWR Pressurized Water Reactor

RCA Reactor Coolant System 